\title{
First Metatarsophalangeal Arthrodesis or Silastic Arthroplasty in the Management of End-Stage Hallux Rigidus: A Comparison of Outcomes Including Proms
}

\section{Eastwood Sarah E*, Kingman Angela, Gray William K, Kakwani Rajesh and Townshend David}

\author{
Northumbria Healthcare NHS Foundation Trust, North Tyneside General Hospital, England
}

*Corresponding author: Miss Sarah E Eastwood, Northumbria Healthcare NHS Foundation Trust, North

Tyneside General Hospital, Rake Lane, North Shields, Tyne and Wear, NE29 8NH, England, Tel: 07815799008

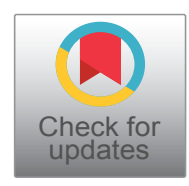

\begin{abstract}
Background: Both first metatarsophalangeal arthrodesis and Swanson silastic arthroplasty are established treatments for the management of end-stage hallux rigidus; identifying the specific outcomes and complications associated with each can represent a challenge when consenting patients.

Methods: PROMs data and clinical outcomes were compared for consecutive series of patients who had received either procedure as surgical management of end-stage hallux rigidus.

Results: There were 61 patients in each group. Patient satisfaction was $87 \%$ following arthrodesis and $73 \%$ following silastic arthroplasty. Over $80 \%$ of patients had an improvement in post-operative PROMs following either procedure. There was a significant difference in mean improvement in favour of arthrodesis (MOXFQ $p=0.0004$, VAS $p=0.002$ ). Metalwork removal was required in $8.2 \%$ of patients following arthrodesis; ongoing pain had been documented in $19.7 \%$ of patients following silastic arthroplasty.

Conclusions: Both procedures remain effective treatment options for end-stage hallux rigidus; local data can be used when counselling patients pre-operatively.
\end{abstract}

\section{Keywords}

Hallux rigidus, Arthrodesis, Silastic arthroplasty, PROMs

\section{Abbreviations}

MTPJ: Metatarsophalangeal Joint; PROMs: Patient Reported Outcome Measures; MOXFQ: Manchester-Oxford Foot Questionnaire; VAS: Visual Analogue Score

\section{Introduction}

The options for the surgical management of end- stage hallux rigidus include arthrodesis, excision interpositional arthroplasty or replacement of the $1^{\text {st }}$ metatarsophalangeal joint (MTPJ) using various different implants. $1^{\text {st }}$ MTPJ arthrodesis has been shown to give good long-term outcomes [1,2] and is widely considered as the "gold standard" against which novel implants are compared [3]. The Swanson double-stemmed silastic arthroplasty was first designed in the 1970's [4]. It has been demonstrated to be an effective treatment with follow-up reported at a mean of 8.5 years in one study [5] and 19 years in another study [6].

The use of patient reported outcome measures (PROMs) is becoming increasingly influential in the strategic planning of orthopaedic surgery in the UK. A commissioning guide for the painful deformed great toe [7] recommends the collection of PROMs data as a standard part of service provision. The Manchester-Oxford Foot Questionnaire (MOXFQ) is a validated outcome tool [8] in foot and ankle surgery whilst the visual analogue score (VAS) is a simple tool recommended for assessing pain [9].

This study compares PROMs data and clinical outcomes between patients who received either a $1^{\text {st }}$ MTPJ arthrodesis or Swanson silastic arthroplasty for management of end-stage hallux rigidus. The aim of the study was to increase the local evidence base to aid and further inform the decision-making process.

\section{Methods}

Surgery was performed under the care of one of 
five consultant Orthopaedic surgeons. There had been a shared-decision-making process between the surgeon and patient to determine the surgical procedure undertaken. Arthrodesis is presented to patients as the current gold standard treatment. In certain patient groups, such as females, particularly those over 60 years of age, silastic arthroplasty may be preferred to maintain some joint function and permit the wearing of high-heeled shoes. The technique for $1^{\text {st }}$ MTPJ arthrodesis varied according to surgeon preference (crossed interfragmentary screws, dorsal plate, dorsal plate with interfragmentary screw). The Swanson double-stemmed hinged silastic arthroplasty without grommets (Swanson flexible hinge toe; Wright Medical Group, N.V.) was used by all surgeons.

In our NHS trust, patients having surgery to the first ray of the foot are routinely invited to complete PROMs questionnaires pre-operatively and at six months post-operatively. Pre-operative questionnaires are completed between the time of listing for surgery and the day of surgery. Post-operative questionnaires are posted to patients with a stamped addressed envelope and an invitation to respond. At both time points this consists of a VAS for pain ( 0 = no pain, $100=$ worse pain) and the MOXFQ. An additional 4-point satisfaction rating is asked at 6 months post-operatively (ratings of Excellent, Good, Fair and Poor). These data are collected, organised and stored with the patients consent, in accordance with Caldicott principles and the Data Protection Act (1998) under local clinical governance approval. A clinical outcomes facilitator is responsible for this.

A consecutive list of patients who had had either $1^{\text {st }}$ MTPJ arthrodesis or $1^{\text {st }}$ MTPJ silastic arthroplasty for the management of hallux rigidus between $1^{\text {st }}$ June 2014 and $30^{\text {th }}$ November 2015 was obtained from clinical coding data and operating theatre logs. Patients whose indication for treatment was not osteoarthritis of the $1^{\text {st }}$ MTPJ and patients undergoing revision arthrodesis procedures were excluded.

Patient demographic data, surgical details and complication rates were obtained by searching electronic patient records. Complications were defined as: superficial or deep infection, re-operation, non-union of arthrodesis, ongoing pain including transfer metatarsalgia and metalwork or implant failure. Patients are generally discharged at three months post-operatively provided there are no ongoing clinical concerns; these patients were not contacted to enquire about further complications. Patients without a satisfactory outcome at 3 months are followed-up until concerns are resolved. If the post-operative PROMs highlight poor outcomes or patient dissatisfaction, then this is brought to the attention of the responsible surgeon and any complications identified from this source were included. Complications were therefore recorded at the latest time-point that the patient had had contact with the department.
Table 1: Basic demographic data of patient series.

\begin{tabular}{|l|l|l|}
\hline Procedure & $\begin{array}{l}\text { st } \text { MTPJ } \\
\text { arthrodesis }\end{array}$ & $\begin{array}{l}\text { Silastic } \\
\text { arthroplasty }\end{array}$ \\
\hline Total number & 61 & 61 \\
\hline Female: Male & $36: 25$ & $52: 9$ \\
\hline Mean age in years (range) & $59.7(42-81)$ & $63.6(30-85)$ \\
\hline Right foot: Left foot & $40: 21$ & $34: 27$ \\
\hline No. complete PROMs (\%) & $36(59.0)$ & $29(47.5)$ \\
\hline
\end{tabular}

For each patient, the difference between post-operative and pre-operative scores for the VAS and MOX$F Q$-index was calculated to determine whether surgery had been beneficial. Mean scores at each time-point and the mean change in score following surgery was compared between the two procedures along with patient satisfaction ratings and complication rates.

Data was collected and analysed using SPSS. Outcome score means were compared using student t-tests and differences between groups analysed using chisquared tests; both with significance of $p<0.05$.

\section{Results}

A total of 122 patients were eligible for inclusion; 61 for each procedure. 88 patients were female, 34 were male. The mean patient age was 61 years (range 30 to 85 years). Pre-operative PROMs data had been obtained in 72 patients (59.0\%), post-operative PROMs data had been obtained in 75 patients (61.5\%) and a complete set of PROMs data had been obtained in 65 patients (53.3\%). Post-operative PROMs data had been collected between 5 and 11 months post-operatively with a mean of 6 months. Post-operative clinical follow-up ranged from 3 months to 2 years with a median follow-up of 6 months. (Table 1) presents the demographic data according to the procedure performed. There was a significant difference between the two groups for gender $\left(x^{2}(1, N=122)=10.44(p=0.001)\right)$ and for age $(t=2.02$, $p=0.046)$.

\section{$1^{\text {st }}$ MTPJ arthrodesis}

Six patients had had previous surgery to the $1^{\text {st }}$ ray; these had all been corrective osteotomies for hallux valgus some years prior to the index surgery.

The complications following arthrodesis were infrequent; there was one episode of superficial infection treated with oral antibiotics and one patient had a failure of arthrodesis requiring revision surgery. Five patients (8.2\%) had required a re-operation for removal of prominent metalwork once fusion had been achieved; these were all prominent interfragmentary screws. One patient (1.6\%) sustained a peri-prosthetic fracture to the proximal phalanx seven months after the index procedure; this was managed non-operatively. Seven patients $(11.5 \%)$ reported symptoms of ongoing pain or transfer metatarsalgia at post-operative review; three of these had been referred to biomechanical podiatry for further management. 
Table 2: PROMs data for $1^{\text {st }}$ MTPJ patients.

\begin{tabular}{|l|l|l|l|l|}
\hline Outcome score & $\begin{array}{l}\text { Number of } \\
\text { patients }\end{array}$ & Minimum & Maximum & Mean \\
\hline Pre-op VAS & 40 & 0 & 95 & 54.6 \\
\hline Post-op VAS & 45 & 0 & 70 & 10.8 \\
\hline $\begin{array}{l}\text { Pre-op MOXFQ- } \\
\text { index }\end{array}$ & 39 & 0 & 94 & 58.6 \\
\hline $\begin{array}{l}\text { Post-op MOXFQ- } \\
\text { index }\end{array}$ & 45 & 0 & 85 & 20.3 \\
\hline
\end{tabular}

Table 3: PROMs data for silastic arthroplasty patients.

\begin{tabular}{|l|l|l|l|l|}
\hline Outcome score & $\begin{array}{l}\text { Number of } \\
\text { patients }\end{array}$ & Minimum & Maximum & Mean \\
\hline Pre-op VAS & 33 & 0 & 100 & 61.0 \\
\hline Post-op VAS & 31 & 0 & 90 & 35.4 \\
\hline $\begin{array}{l}\text { Pre-op MOXFQ- } \\
\text { index }\end{array}$ & 33 & 25 & 96 & 61.5 \\
\hline $\begin{array}{l}\text { Post-op MOXFQ- } \\
\text { index }\end{array}$ & 31 & 0 & 91 & 44.0 \\
\hline
\end{tabular}

Table 4: Comparative table of mean pre-operative PROMs scores; analysis of difference using t-tests.

\begin{tabular}{|l|l|l|l|}
\hline & Arthrodesis $(\mathbf{n}=\mathbf{4 0})$ & Silastic arthroplasty $(\mathbf{n}=\mathbf{3 3})$ & p-value \\
\hline Mean pre-op VAS (SD) & $54.6(27.4)$ & $61.0(22.9)$ & 0.280 \\
\hline Mean pre-op MOXFQ (SD) & $58.6(23.3)$ & $61.5(17.8)$ & 0.561 \\
\hline
\end{tabular}

Table 5: Mean change in VAS and MOXFQ after arthrodesis or silastic arthroplasty and analysis using t-tests.

\begin{tabular}{|c|c|c|c|c|c|c|}
\hline & & $\begin{array}{l}\text { Mean pre-op } \\
\text { score (SD) }\end{array}$ & $\begin{array}{l}\text { Mean post-op } \\
\text { score (SD) }\end{array}$ & $\begin{array}{l}\text { Mean change in } \\
\text { score (SD) }\end{array}$ & p-value & $\begin{array}{l}\text { Arthrodesis vs. silastic } \\
\text { arthroplasty }\end{array}$ \\
\hline \multirow[t]{2}{*}{ VAS } & Arthrodesis $(n=36)$ & $55(26.9)$ & $11(17.0)$ & $44(29.1)$ & $<0.0001$ & \multirow[t]{2}{*}{${ }^{*} p=0.002$} \\
\hline & $\begin{array}{l}\text { Silastic } \\
\text { arthroplasty }(n=29)\end{array}$ & $62(20.5)$ & $38(27.7)$ & $24(20.5)$ & 0.0004 & \\
\hline \multirow[t]{3}{*}{ MOXFQ } & Arthrodesis $(n=36)$ & $59(22.8)$ & $20(20.2)$ & $38(21.9)$ & $<0.0001$ & \multirow[t]{2}{*}{${ }^{*} p=0.0004$} \\
\hline & $\begin{array}{l}\text { Silastic arthroplasty } \\
(n=29)\end{array}$ & $62(17.5)$ & $44(27.6)$ & $18(21.2)$ & 0.005 & \\
\hline & & & & \multicolumn{3}{|c|}{$\begin{array}{l}\text { *Greater improvement after arthrodesis than silastic } \\
\text { arthroplasty }\end{array}$} \\
\hline
\end{tabular}

The PROMs data are summarised in (Table 2). Both pre-operative and post-operative PROMs data were present for 36 patients. An improvement in VAS post-operatively was seen in 33 patients $(91.7 \%)$ with an overall mean change of -44.5 points (SD 29.1, range 0-95 point improvement). An improvement in MOXFQ score post-operatively was seen in 34 patients (94.4\%) with an overall mean change in MOXFQ of -38.7 points (SD 21.9, range 0-91 point improvement).

\section{Silastic arthroplasty}

Of the 61 patients who had received a silastic arthroplasty, seven patients had had previous surgery of the $1^{\text {st }}$ ray (cheilectomy or corrective osteotomy procedure).

Complication rates were also low following silastic arthroplasty. One patient (1.6\%) required antibiotics for a superficial wound infection, there were no cases of deep infection. Further surgery was required in one patient (1.6\%) who had persistent swelling and pain and a decision was made to revise to an arthrodesis. Twelve patients (19.7\%) had reported symptoms of ongoing pain or transfer metatarsalgia, of these 5 had been referred to biomechanical podiatry for further management.

The PROMs data are summarised in Table 3. Both pre-operative and post-operative PROMs data were present for 29 patients. An improvement in VAS post-operatively was seen in 24 patients (82.8\%) with an overall mean change of -24.4 points (SD 20.5, range 10-point worsening to 70 point improvement). An improvement in MOXFQ score post-operatively was seen in 25 pa- tients (86.2\%) with an overall mean change in MOXFQ of -18.0 points (SD 21.2, range 36 point worsening to 55 point improvement).

\section{Comparative statistics}

There was no significant difference in the proportion of complete PROMs data returned between the two procedures, $\left(\chi^{2}(1, N=122)=1.61(p=0.204)\right)$. There was no significant difference in mean pre-operative scores for either VAS $(t=1.08, p=0.284$ ) or MOXFQ ( $t=0.583, p=$ 0.561 ) between the two procedures as shown in Table 4. Whilst there was an overall mean improvement in both VAS and MOXFQ following both arthrodesis and silastic arthroplasty, the mean improvement was greater after arthrodesis as shown in Table 5. These differences remained significant when adjusted for gender and age (bivariate analysis; VAS $p=0.009$, MOXFQ $p=0.001$ ).

Patient satisfaction scores following surgery are demonstrated in figure one. Following $1^{\text {st }}$ MTPJ arthrodesis, $87 \%$ of patients had rated their surgery as "excellent" or "good" whilst $73 \%$ had attributed the same rating after silastic arthroplasty. The observed higher satisfaction rating following arthrodesis was not statistically significant $\left(\chi^{2}(1, N=65)=3.08(p=0.079)\right)$ (Figure 1$)$.

Complication rates were generally low for both procedures and a comparison is seen in (Table 6). Transfer metatarsalgia or ongoing pain was seen following both procedures and there was no statistically significant difference in its incidence between the two procedures, $\left(\chi^{2}(1, N=122)=0.997(p=0.318)\right)$. 
Table 6: Incidence of complications following the two procedures.

\section{Complication}

Post-operative infection (\%)

Non-union (\%)

Revision procedure (\%)

Metalwork removal (\%)

Ongoing pain/transfer metatarsalgia (\%)

$1^{\text {st }}$ MTPJ arthrodesis

$1(1.6)$

$1(1.6)$

$1(1.6)$

$5(8.2)$

7 (11.5)
Silastic arthroplasty

1 (1.6)

$1(1.6)$

$12(19.7)$

\section{Patient rating of surgery post-operatively}

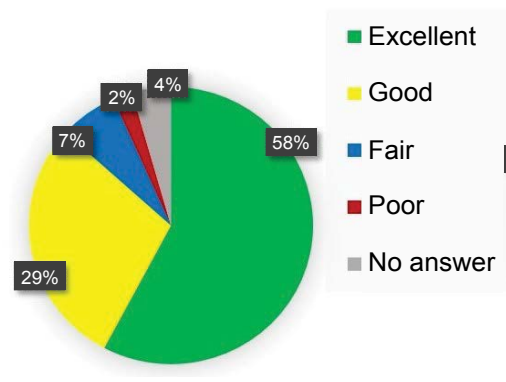

Arthrodesis

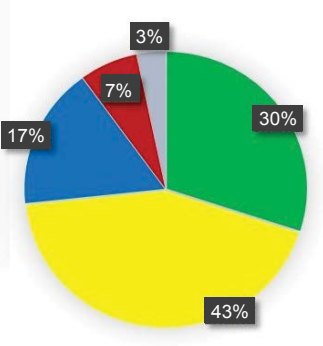

Silastic arthroplasty
Figure 1: Patient satisfaction rating score post-operatively according to procedure performed (arthrodesis $n=45$, silastic arthroplasty $n=30$ ).

\section{Discussion}

$1^{\text {st }}$ MTPJ arthrodesis and silastic arthroplasty are both established surgical treatments for end-stage hallux rigidus. This retrospective comparative case series has examined the outcomes of both within an NHS trust. It has shown that the majority of patients have an improvement in function and reduction in pain at six months post-operatively following either procedure. The majority of patients were satisfied following either procedure.

Following arthrodesis, an improvement in both VAS and MOXFQ was observed in 33 out of 36 patients for whom data were available. Goucher and Coughlin [10] reported similar patient satisfaction rates following arthrodesis to the current study. Re-operation rate following arthrodesis was $9.8 \%$ in the current series; this was mostly removal of prominent metalwork. One concern regarding arthrodesis is the rate of non-union; the incidence of $1.6 \%$ in this series is comparable with the rate of symptomatic non-union reported in a large systematic review [11]. There was only one re-operation in the silastic arthroplasty group which is in comparison with other series that have reported low re-operation rates [6] in conjunction with good patient satisfaction [5]. In the current study, follow-up period was relatively short so long-term re-operation rates and patient satisfaction following these procedures is not known. Ongoing pain or a diagnosis of transfer metatarsalgia had been documented in $19.7 \%$ of patients following silastic arthroplasty. This is something which requires further study to be better understood; one study has previously reported that in $46.7 \%$ of patients following silastic arthroplasty the maximum pressure measured with pedobarog- raphy lies under the lesser metatarsals rather than the first metatarsal [5].

\section{Strengths and limitations}

This is a review of current practice and therefore neither surgical technique nor post-operative regimen was standardised. The only exclusion criteria were surgery for a reason other than osteoarthritis of the joint or revision surgery. The study included all patients identified retrospectively through clinical coding and operative logs as having had these procedures; this relied on those logs being completed accurately. There was no randomisation of patients, instead the surgical procedure had been selected through an informed shared decision-making process which will be influenced by surgeon opinion and patient factors. This might contribute to the gender imbalance observed between the procedures with a predominantly female population having silastic arthroplasty. The comparisons that can be made between outcomes and complication rates for two procedures are therefore limited. Patients were not stratified based on co-morbidities which may also influence surgical choice. This series has however highlighted the rates of specific complications associated with each procedure in our unit and these can now be discussed with patients as part of the informed consent process.

A further limitation of this work is the lack of complete PROMs data. There was an overall response rate of $53.3 \%$ and a complete set of PROMs data in only $47.5 \%$ of patients who had received silastic arthroplasty. It is recognised that collection of PROMs data has difficulties and can introduce bias to results [12]. Post-operative PROMs questionnaires had been returned by patients with and without post-operative complications and across a range of outcomes. Various measures have been introduced in our institution to increase the response rate to PROMs; this work is ongoing and will be further analysed.

\section{Conclusion}

This comparative case series demonstrates that both arthrodesis and silastic arthroplasty remain effective treatments for the management of end-stage hallux rigidus. As newer arthroplasty implants are developed [3]; these established treatments should not be forgotten. Local PROMs and clinical outcomes data can be utilised when counselling patients in a shared decision-making process. Further robust evidence with matched patient cohorts, particularly in-patient groups where there is clinical equipoise, is recommended to compare lon- 
ger-term outcomes and the healthcare economics associated with these procedures.

\section{Acknowledgements}

No external funding was received for this work.

\section{Conflict of Interest}

On behalf of all authors, the corresponding author states that there is no conflict of interest.

\section{References}

1. Fitzgerald JA, Wilkinson JM (1981) Arthrodesis of the metatarsophalangeal joint of the great toe. Clinical Orthopaedics and Related Research 157: 70-77.

2. Gibson JA, Thomson CE (2005) Arthrodesis or total replacement arthroplasty for hallux rigidus: A randomized controlled trial. Foot Ankle Int 26: 680-690.

3. Daniels TR, Younger AS, Penner MJ, Wing KJ, Miniaci Coxhead SL, et al. (2016) Midterm outcomes of polyvinyl alcohol hydrogel hemiarthroplasty of the first metatarsophalangeal joint in advanced hallux rigidus. Foot Ankle Int 38: 243-247.

4. Swanson AB (1972) Implant arthroplasty for the great toe. Clin Orthop Relat Res 85: 75-81.

5. Morgan S, Ng A, Clough T (2012) The long-term outcome of silastic implant arthroplasty of the first metatarsophalangeal joint: A retrospective analysis of one hundred and eight feet. Int Orthop 36: 1865-1869.

6. van Duijvenbode DC, Bulstra GH, Nijsse BA (2013) Nineteen-year follow-up of the silastic double stemmed hinge prosthesis of the first metatarsophalangeal joint. Foot Ankle Surg 19: 27-30.

7. BOFAS, BOA, RCSEng, (2013) Commissioning guide: Painful deformed great toe in adults. 2-22.

8. Dawson J, Doll H, Coffey J, Jenkinson C (2007) Responsiveness and minimally important change for the Manchester-Oxford foot questionnaire (MOXFQ) compared with AOFAS and SF-36 assessments following surgery for hallux valgus. OsteoArthritis Cartilage 15: 918-931.

9. Schrier JC, Palmen LN, Verheyen CC, Jansen J, Koëter S (2015) Patient-reported outcome measures in hallux valgus surgery. A review of literature. Foot Ankle Surg 21: 11-15.

10. Goucher NR, Coughlin MJ (2006) Hallux metatarsophalangeal joint arthrodesis using dome-shaped reamers and dorsal plate fixation: A prospective study. Foot Ankle Int 27: 869-876.

11. Roukis TS (2011) Nonunion after arthrodesis of the first metatarsal-phalangeal joint: A systematic review. J Foot Ankle Surg 50: 710-713.

12. Hutchings A, Neuburger J, Frie KG, Black N, van der Meulen $\mathrm{J}$ (2012) Factors associated with non-response in routine use of patient reported outcome measures after elective surgery in England. Health Qual Life Outcomes 10: 34. 